\title{
Silent plaque rupture in the left main stem assessed by optical coherence tomography
}

\author{
Yongcheol Kim ${ }^{1}$, Byeong-Keuk Kim², Thomas W. Johnson ${ }^{3}$, Sung-Jin Hong ${ }^{2}$, \\ Chul-Min $\mathrm{Ahn}^{2}$, Jung-Sun Kim ${ }^{2}$, Deok-Kyu Cho', Young-Guk Ko ${ }^{2}$, \\ Donghoon Choi ${ }^{1}$, Myeong-Ki Hong ${ }^{2}$, Yangsoo Jang ${ }^{2}$ \\ ${ }^{1}$ Division of Cardiology, Department of Internal Medicine, Yonsei University College of Medicine \\ and Cardiovascular Center, Yongin Severance Hospital, Yongin, Republic of Korea \\ ${ }^{2}$ Division of Cardiology, Severance Cardiovascular Hospital, \\ Yonsei University College of Medicine, Seoul, Republic of Korea \\ ${ }^{3}$ Department of Cardiology, Bristol Heart Institute, Bristol, United Kingdom
}

A 59-year-old man underwent primary percutaneous coronary intervention (PCI) for thrombotic total occlusion of his distal right coronary artery. Bystander disease was limited to an ambiguous lesion in the distal left main stem (LMS) with an intraluminal filling defect suggestive of a ruptured plaque. A staged inpatient assessment of the LMS was undertaken utilizing invasive physiologic and intravascular imaging assessments as distal flow in the left coronary system was preserved and symptoms had settled with index intervention (Fig. 1A, B; Suppl. Video 1 and 2). Evaluation of the LMS lesion by instantaneous wave-free ratio (iFR) and fractional flow reserve (FFR), 3 days following presentation, provided an iFR value of 0.95 and the hyperemic FFR value was 0.89 . Optical coherence tomography (OCT) demonstrated a ruptured plaque without associated thrombus in the LMS with diffuse fibroatheroma with underlying necrotic core extending into the proximal left anterior descending artery (LAD) (Fig. 1C-F, Suppl. Video 3). Importantly, OCT generated a minimal lumen area (MLA) in the LMS of $8.73 \mathrm{~mm}^{2}$ (Fig. 1G), with a MLA of $3.04 \mathrm{~mm}^{2}$ in the proximal LAD segment. Therefore, based on the findings of the iFR/FFR and OCT assessment, it was concluded that the LMS plaque had stabilized following a previous rupture and in the absence of ischemia, elected to pursue a conservative strategy. At 3-month follow-up a treadmill test demonstrated good exercise tolerance (11.2 metabolic equivalents) without symptoms or electrocardiographic evidence of ischemia.

Although an acute phase of rupture plaque is defined by a disrupted fibrous cap, with or without thrombus, overlying a necrotic core, a definition of the chronic phase has, to date, not been well described [1]. The OCT is more accurate for detecting plaque characteristics due to its higher resolution, which is 10 times $(10 \mu \mathrm{m})$ that of intravascular ultrasound [2]. The present case highlights the utility of OCT in the evaluation of ambiguous lesions, with the use of MLA to guide LMS intervention and detection of a smooth-edged, disrupted plaque devoid of thrombus and a necrotic core, is suggestive of a stabilized healed ruptured plaque.

\section{Conflict of interest: None declared}

\section{References}

1. Johnson TW, Räber L, di Mario C, et al. Clinical use of intracoronary imaging. Part 2 : acute coronary syndromes, ambiguous coronary angiography findings, and guiding interventional decision-making: an expert consensus document of the European Association of Percutaneous Cardiovascular Interventions. Eur Heart J. 2019; 40(31): 2566-2584, doi: 10.1093/eurheartj/ehz332, indexed in Pubmed: 31112213.

2. Kim Y, Johnson TW, Akasaka T, et al. The role of optical coherence tomography in the setting of acute myocardial infarction. J Cardiol. 2018; 72(3): 186-192, doi: 10.1016/j.jjcc.2018.03.004, indexed in Pubmed: 29650398.

Address for correspondence: Byeong-Keuk Kim, MD, PhD, Division of Cardiology, Severance Cardiovascular Hospital, Yonsei University College of Medicine, 250 Seongsan-ro, Seodaemun-gu, Seoul 03722, Republic of Korea, tel: +82-2-2228-8460, fax: +82-2-2227-7732, e-mail: kimbk@yuhs.ac

Received: 16.03 .2020

Accepted: 26.03.2020 


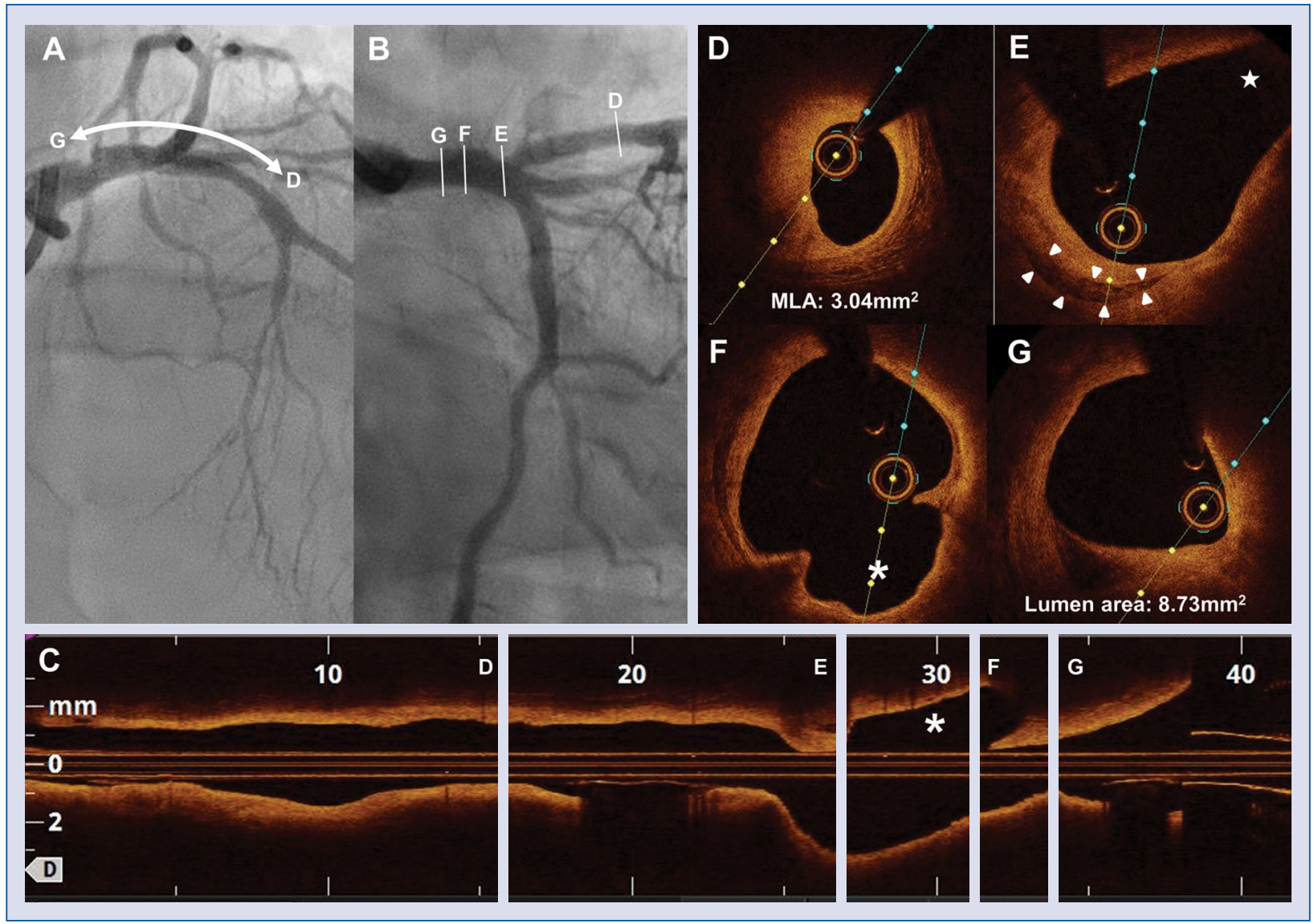

Figure 1. A, B. Angiographic assessment demonstrating mild stenosis with suspicious ruptured plaque in the left main stem (LMS); C. Longitudinal optical coherence tomography (OCT) imaging demonstrating a ruptured plaque space (asterisk); D. Minimal lumen area (MLA) of $3.04 \mathrm{~mm}^{2}$ in the proximal left anterior descending artery; E. OCT, showing suspicious necrotic core (arrowheads) in the left main bifurcation (star: ostium of left circumflex artery; F. OCT demonstrating ruptured plaque space in the LMS; G. MLA of $8.73 \mathrm{~mm}^{2}$ in the LMS. 